\title{
Software Development Model of Information System
}

\author{
Shiyun Sun \\ Yunnan Technology and Business University, Yunnan, Kunming, 650000, China
}

Keywords: Information system, Software development, Model.

\begin{abstract}
With the continuous development of information technology and science and technology, software development management model consists of four stages, host terminal, file server, C/S, and $\mathrm{B} / \mathrm{S}$. The first two software development models have been phased out of history, and the latter two models have been widely used in software management, and have their own advantages. This paper mainly analyzes information system software development models.
\end{abstract}

\section{Introduction}

In recent years, with the continuous change of management software development model, now market competition appears increasingly fierce, and software companies constantly update technology and management models, in order to be able to develop software to meet market demand, to ensure that companies have competitive advantages.

\section{History of Information Software Development Model}

\section{Host terminal model.}

In the 1960s and 1970s, host terminal model in software development occupied the leading position. The host terminal model includes two kinds of components: client and host. The host terminal has advantages of centralized management and good safety, but its defect is expensive price, and databases and applications are generally in the mainframe, leading to irrational division of application logic, therefore, it could not reasonably save the hardware investment and then was gradually phased out.

\section{File server model.}

In the 1980s, personal computer appeared in business. When personal computer was applied in business, LAN also emerged, and new idea of file server forms. In file server, multiple central servers provide ways for access to information resources. This model has flexible configuration and low cost, but also runs application program in client PC, and the file server can only provide the system with file services. This way is suitable for application in small-scale LAN. Based on the above, two models were gradually eliminated by the society, and then $\mathrm{B} / \mathrm{S}$ and $\mathrm{C} / \mathrm{S}$ software structure models were formed.

\section{C/S model.}

In the 1980s, C/S model was gradually formed. In C/S software development structure, network computer is divided into two parts, server and client, and the client is basically a PC with general function, to ensure rational use of server resources. C/S software structure is suitable for application in LAN without too many users. At this stage, most financial software uses C/S structure. In order to form the corresponding process, C/S software structure needs two separate entities, server and client. The client must issue a request to the server, and then the server provides service. For example, use SQL query for requests, establish SQL database engine, and then use the above-described engine for reasonably processing system request, and finally form the result set. Based on this, two independent processes collaborate to accomplish common tasks. If the client is able to meet customer requests and will directly form results, or require the server to handle user requests. If invoking the public data on the server, the server processes and then sends to the customer. C/S software structure model requires reasonably handling and balancing things, to ensure consistent and complete data. 


\section{1) Advantages of $C / S$ software}

First, the server application is provided with relatively light running load. The simplest C/S structure includes the database server and client applications, also known as background program and foreground program. The database server operation machine is the application server. If in need to operate the database, the customer can set automatic research, and then send a certain request, in accordance with rules of reservation, the server responds, and then outputs the result, resulting in relatively light server running data.

Second, it is provided with transparent storage management of server. When in use, the database has appropriate storage and management function. In fact, the client application program and server program run separately, but the foreground application is not consistent with the actual provisions, and the general server program cannot present different operational data in a central way, such as, repeated number and visitor permissions. Accordingly, the transparent foreground program on work surface can complete the work without analysis and interference on background program.

2) Disadvantages of C/S software

$\mathrm{C} / \mathrm{S}$ software has relatively higher operating and maintenance cost. First, in application of C/S software in system, the system should use a reasonable database platform to unify the data source of database, and then the database can manage synchronously data information at two places, but in the actual operation, simultaneous access data at two places cannot be achieved. If the system needs to establish a real-time database, between the two places real-time communication needs to be built, and online running of database server at two places also needs to be guaranteed. Therefore, relevant operators need to maintain not only database management server, but also client management server, which requires high technology cost and maintenance cost. Second, C/S traditional structure needs to operate and develop different versions, because product upgrading is faster, and low efficiency and high cost cannot meet the actual demand. After JAVA platform appeared, B/S platform seriously challenged C/S platform. Different clients require installing different software, and user interfaces of different sizes, resulting in more complex software platform. Because different users install different programs, it is difficult and complex to upgrade and maintain, and if you want to upgrade, you need to constantly update the software for all users ${ }^{[1]}$.

\section{B/S model.}

With the development and progress of information technology and computer network technology, $\mathrm{B} / \mathrm{S}$ structure was formed after continuous improvement of C/S structure. B/S structure can fully demonstrate the software application business logic in server terminal, and also reflect on the performance of users on Web. Clients only need to handle the browser. It is an emerging software technology and is also the main structure of future application software. B/S structure consists of three levels, database server, Web server and browser. B/S structure client uses a common browser instead of the conventional application software, and the user uses the browser for all operations. The key of $\mathrm{B} / \mathrm{S}$ structure is Web server, able to accept remote query request, obtain information in the database based on the query specification, then form a variety of page languages and HTML language, and finally send back to the browser. In addition, the browser also needs to promptly delete and change data information. Propose requests on Web server, and database and browser together complete the work.

\section{1) Advantages of $B / S$ software}

First, it is easy to upgrade and maintain. At this stage, for improvement and upgrading, software system need frequent upgrading. B/S structure is obviously easy to maintain and upgrade. No matter what kind of system in which it is applied, it will not increase the workload of system upgrading and maintenance. The user only needs to maintain and upgrade the server, which can save manpower and material resources to a great extent.

Second, it has low cost and multiple selections. Computer system is basically Windows system, and the standard configuration is the browser, but Windows is not the absolute ruler in the server. At present, when management software uses B/S structure, it only needs to be installed on a Linux server, with relatively higher security, therefore, in the system there will be a lot of choices, and Windows desktop operating system will not be affected by the system type. Because B/S structure is only 
installed on the server, managers only need to manage the server. The user interface uses WWW browser to achieve business logic, and just a small part achieves business logic at the foreground, therefore, managers only need to maintain and manage the browse ${ }^{[2]}$.

\section{2) Disadvantages of $B / S$ software}

Data running load in application server is relatively heavy. The server needs to handle query requirements, and then based on page forms deliver the result to the browser. The server generally has a relatively large load, and requires good performance. Web server provides all page data, and after the completion of handling, also needs network transmission, resulting in relatively long time for display.

\section{Comparative Analysis on B/S and C/S}

First, safety comparison. Hackers, viruses, earthquakes, fires, and others are main security threats for data. In addition, group-level remote software, needs to install multiple C/S structure servers, and synchronize the data of multiple servers, which will affect the security of data points, and then affect the overall security of the data. But B/S structure software system can centrally store the database server, without the need of the client connecting to the database and business data, or the need to synchronize data, so there are no data security issues.

Second, data consistency comparison. C/S structure system handles off-site running group data according to installation ways of servers in various areas, and then synchronizes data. Access to data through this model is relatively slow. B/S software structure system centrally stores data, and central data bureau can always check single business data on client terminal, without data inconsistencies.

Third, data real-time property comparison. When group application program uses C/S structure, it cannot at any time check the current business situation, while when using B/S structure, group application program can not only check the current service, and also avoid unnecessary loss of business ${ }^{[3]}$.

Fourth, data traceability comparison. B/S structure can store data centrally, therefore, headquarters can directly trace original business data document of branches. However, when C/S structure does not have this property described above. In order to reduce the amount of transmissions, if just uploading the intermediate data, headquarters cannot check the original documents in time.

Fifth, service response timeliness comparison. C/S structure software forms distributed application, and needs to install all node programs, so even if there are just minor flaws, it needs to be redeployed. To protect the system consistency, in case of redeployment, it is necessary to stop the updating service. B/S structure at headquarters server centralizes data, without the need of any node, namely, just updating one node, then updating all programs, able to quickly reflect service data.

Sixth, network application restriction comparison. C/S structure is suitable for application in bandwidth users or LAN, but B/S structure can be applied in any network, especially suitable for the area where the bandwidth cannot be installed.

\section{Combined Application of B/S and C/S and Complementary Advantages}

After comparative analysis of $\mathrm{C} / \mathrm{S}$ model and B/S model, combine the advantages of both software models, to create combined application model. In many large-scale information systems, the majority of users use B/S software structure. After the browser software is used on the client computer, the server with higher performance summarizes and stores basic data, and at the interval it is necessary to build Web server, as the exchange channel between client browser and data server. Information system has relatively strong interaction, high security, and large data processing and other advantages, but also needs to have beautiful interface, flexible data query and complex site functions. Therefore, based on this information system, it is necessary to rationally use C/S model. This combined way can not only guarantee to fully reflect characteristics and advantages of two models, and develop high-efficiency, low-cost, convenient and flexible, reliable and secure software systems. ${ }^{[4]}$ 
At this stage, in the market, $\mathrm{C} / \mathrm{S}$ model and B/S mod software products have their own advantages. When small and mediate enterprises establish information management systems, because $\mathrm{C} / \mathrm{S}$ software model has the beautiful interface, cheap cost and other advantage, it gets favored by small and medium enterprises, and occupies the dominant position. However, when establishing information systems in cross-regional and large-scale enterprises, B/S software model has obvious advantages, and gradually develops into an important software model. Domestic leading enterprise management software enterprise, UFIDA Software Company, and the world's top software business management maker, SAP, both rationally use C/S model and B/S model software. At this stage, enterprises need to establish different sales methods according to different market demands, to promote common development and progress of $\mathrm{C} / \mathrm{S}$ model and $\mathrm{B} / \mathrm{S}$ model. Then enterprises can get more and larger market shares and competitive advantages.

With the continuous development of information technology and computer network technology, all kinds of information system software are gradually improved. File server, host terminal and other traditional software models have gradually been eliminated, and at present, mainstream models in software market are $\mathrm{C} / \mathrm{S}$ and $\mathrm{B} / \mathrm{S}$ model. In $\mathrm{C} / \mathrm{S}$ software structure, compared to two-layer structure, three-layer structure has many advantages, and gradually replaces two-layer software structure. C/S and B/S software structure models have their advantages and defects, cannot replace each other, and will co-exist in software market in the future for a long period. B/S products have higher prices compared to C/S products. B/S software structure model is suitable for application in cross-regional and large enterprises, while $\mathrm{C} / \mathrm{S}$ software structure model is suitable for application in small and medium enterprises ${ }^{[5]}$.

\section{Conclusions}

To sum up, in recent years, with the continuous improvement and development of information technology, science and technology, and computer network technology, information system software development gradually phased out traditional file server, and host terminal model, and established C/ $\mathrm{S}$ and B/S model software products, in order to further improve the quality and efficiency of the management of enterprise software. This paper analyzes the history of information system software development, and makes a comparative analysis on C/S model system application method and B/S software development system to ensure to be able to comprehensively improve economic efficiency of enterprises.

\section{References}

[1] Jia Suling, Wang Qiang, Wang Hongsen. Management Information System, Machinery Industry Press, 2014, 9: 77-82.

[2] Peng Yuan. Large-scale Distributed Systems Architecture and Design, Machinery Industry Press, 2014, 2: 44-45,37.

[3] Yang Xueqiang, Huang Jing, Bai Yong, et. Component Based Equipment Management Integrated Information System Software Development, Journal of Armored Force Engineering College, 2012, 26 (2): 73-76.

[4] Zhang Rong, Xiao Dongmei, Liu Congming, et. Application of Transportation Scheduling Information System in Medical Transportation Management, Journal of Nursing Science, 2013, 28 (18): 12-13.

[5] Wang Gang, Zhang Yuanyuan, He Gang. Analysis and Research on Postal Savings Bank Information System Development Project Quality Management, Henan Science and Technology, 2013 (17): 7-9. 